\title{
Reversible Magnetization of Melt-Processed NEG-123 Superconductor
}

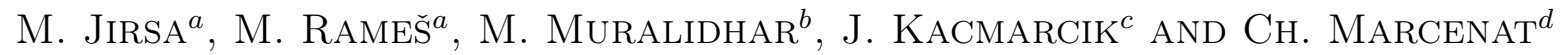 \\ ${ }^{a}$ Institute of Physics, ASCR, v.v.i., Na Slovance 2, CZ-18221 Praha 8, Czech Republic \\ ${ }^{b}$ Railway Technical Research Institute, 2-8-38 Hikari-cho, Kokubuni-shi, Tokyo 185-8540, Japan \\ ${ }^{c}$ Institute of Experimental Physics SAS, Kosice, Slovakia \\ ${ }^{d}$ CEA Grenoble, Grenoble Cedex 9, France
}

\begin{abstract}
Specific heat of superconducting electrons was measured in a melt-processed $\left(\mathrm{Nd}_{0.33} \mathrm{Eu}_{0.38} \mathrm{Gd}_{0.28}\right) \mathrm{Ba}_{2} \mathrm{Cu}_{3} \mathrm{O}_{y}$ superconductor (NEG-123) with $5 \mathrm{~mol} \%$ of Gd-211, $1 \mathrm{wt} \% \mathrm{Ag}$, and $0.035 \mathrm{~mol} \%$ of Zn. The thermodynamic characteristics of this compound are still not well known. From calorimetric measurements the reversible magnetization, critical fields, penetration depth, and coherence length were deduced. In the vicinity of $T_{\mathrm{c}}$, the reversible magnetic moment was additionally measured by means of SQUID.
\end{abstract}

PACS numbers: 74.25.Ha, 74.25.Bt, 74.25.Op

\section{Introduction}

The LRE-123 (LRE = light rare earth, LRE $=\mathrm{Nd}$, Sm, $\mathrm{Eu}, \mathrm{Gd}$ ) materials exhibit exceptional pinning properties partly due to point-like LRE/Ba solid solution clusters, partly for the introduced nanoscopic secondary phase particles. As the LRE/Ba clusters substitute the role of oxygen vacancies, representing the typical point-like defects in bulk RE-123 compounds, the LRE-123 compounds can be optimally oxygenated to reach the highest possible $T_{\mathrm{c}}$ together with a high secondary peak. A high remnant critical current density is due to secondary phase nanoparticles. The $\left(\mathrm{Nd}_{0.33} \mathrm{Eu}_{0.38} \mathrm{Gd}_{0.28}\right) \mathrm{Ba}_{2} \mathrm{Cu}_{3} \mathrm{O}_{y}$ compound studied here exhibits one more distinguished type of defects, nanoscale lamellas filling channels between regular twin boundaries. The aim of this work is for the first time to look at the thermodynamic properties of this interesting material.

The sample in size of $0.7 \times 0.7 \times 0.4 \mathrm{~mm}^{3}$ was cut from a pellet of $\left(\mathrm{Nd}_{0.33} \mathrm{Eu}_{0.38} \mathrm{Gd}_{0.28}\right) \mathrm{Ba}_{2} \mathrm{Cu}_{3} \mathrm{O}_{y}$ doped with $5 \mathrm{~mol} \% \mathrm{Gd}-211,1$ wt\% Ag, $0.035 \mathrm{~mol} \%$ of $\mathrm{ZnO}$, and $0.5 \mathrm{~mol} \% \mathrm{Pt}$ [1]. The pellet, prepared by the oxygen-controlled-melt-growth (OCMG) process in $\mathrm{Ar} / 0.1 \% \mathrm{O}_{2}$, had $16 \mathrm{~mm}$ in diameter and was $8 \mathrm{~mm}$ high. $T_{\text {c }}$ reached $92.8 \mathrm{~K}$.

\section{Results and discussion}

The differential specific heat measurement, presented in terms of $C(T) / T$, was measured in the temperature range 73 to $99.3 \mathrm{~K}$, in a series of constant magnetic fields $B=\mu_{0} H=0,1,2,3,5$, and $7 \mathrm{~T}$. The measured curves are seen in Fig. 1a. With the aim to evaluate the reversible magnetization, the data were processed with the help of the following thermodynamic relations [2]:

$$
\Delta S(B, T)=-\int_{T^{*}}^{T} \frac{\Delta C(B, T)}{T} \mathrm{~d} T
$$

and

$$
\Delta F(B, T)=-\int_{T^{*}}^{T} \frac{\Delta S(B, T)}{T} \mathrm{~d} T,
$$

where $\Delta C(B, T)=C(B, T)-C(0, T)$ (see Fig. 1b), $\Delta S$ is entropy and $\Delta F$ is free energy of the system. $T^{*}$ is the highest measured temperature, $T^{*}=99.3 \mathrm{~K}$.

The $\Delta F(T)_{B}$ results obtained by the integration of Eq. (1) are shown in Fig. 1b. As the original experimental data were not calibrated, all the following data processing gives only relative quantities. Reversible magnetization comes from $M_{\mathrm{rev}}(B, T)=-(\partial \Delta F(B, T) / \partial B)_{T}$. At higher magnetic fields $M_{\mathrm{rev}}(B)$ should obey the functional dependence $M_{\mathrm{rev}}(B, T)=-\alpha+\beta \ln B$. We used the integral function to this dependence, $\gamma-(\alpha+\beta) B+$ $\beta B \ln B$, where $\gamma$ is integration constant, to fit directly the $\Delta F(B)$ data. These data were obtained from vertical cuts at a series of temperatures, with $1 \mathrm{~K}$ step (indicated by dashed lines in Fig. 1b) in the system of five $\Delta F(T)_{B}$ curves for different fields. The $\Delta F(B)_{T}$ dependences (all going to $(0,0))$ are shown in Fig. 1c, together with their fits (lines). The parameters $\alpha$ and $\beta$ obtained in this way enabled us to construct the $M_{\mathrm{rev}}(B)$ dependences presented in Fig. 2a. As indicated above, the data were obtained in arbitrary units. An indicative scale, provided on the right side of the figure, was obtained from direct measurements of reversible magnetization by SQUID $(\mathrm{RM})$. It is seen that the reversible magnetization data in vicinity of $T_{\mathrm{c}}$ lie deep below 1 Oe $(\approx 80 \mathrm{~A} / \mathrm{m})$. Any direct experimental determination of such curves (SQUID, 

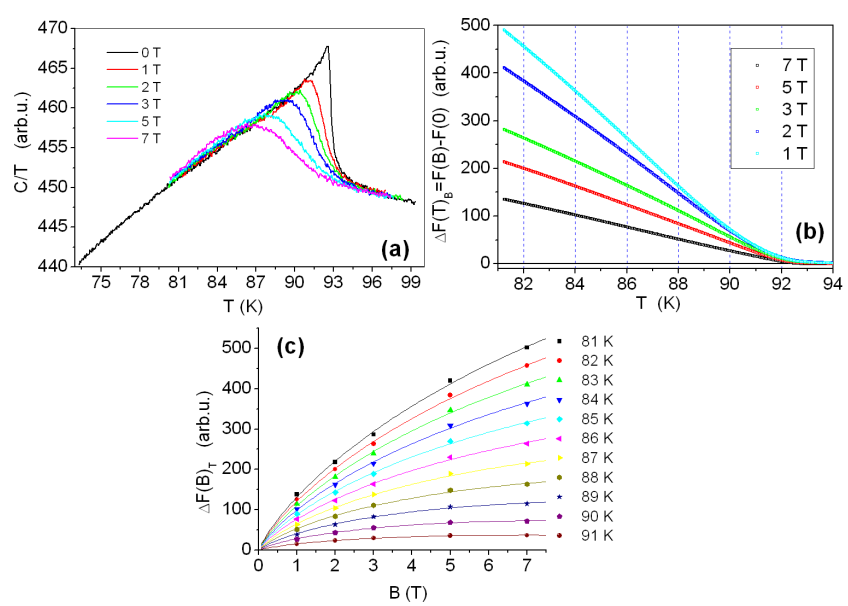

Fig. 1. The temperature dependences of (a) $C(B, T) / T$, (b) the free energy difference as a function of temperature, as obtained from the integration in Eq. (1), and (c) the same data as in (b), however transferred to the $\Delta F(B)_{T}$ representation (symbols) and fitted by the functional dependence $\gamma-(\alpha+\beta) B+\beta B \ln B$ (lines).
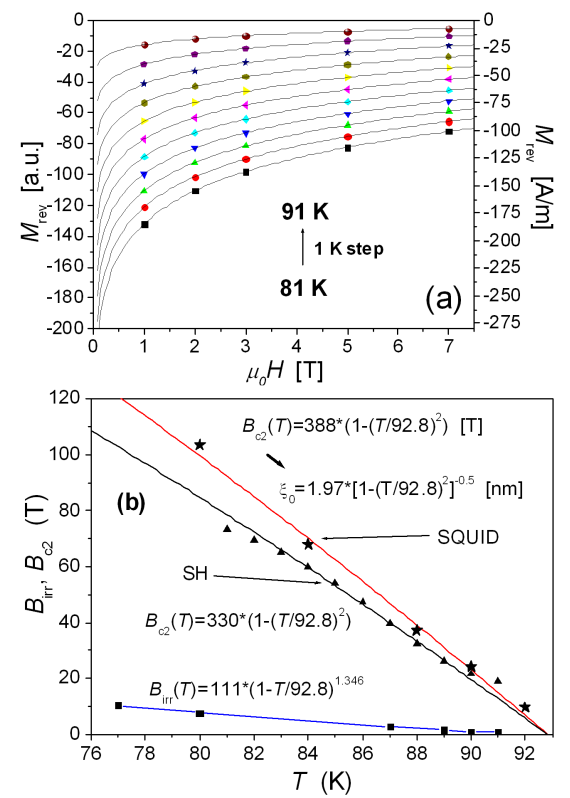

Fig. 2. (a) $M_{\text {rev }}(B)$ constructed from the fits of Fig. 1c; (b) temperature dependences of $B_{\mathrm{c} 2}$ obtained from $\mathrm{CP}$ and SQUID measurements and $B_{\mathrm{irr}}(T)$ from independent irreversible vibrating sample magnetometer (VSM) experiment.
VSM) is then associated with a high experimental error.

Results of the CP evaluation are shown in Fig. 2b, together with the direct reversible magnetization measured by SQUID. The lines are fits to common theoretical formulae. Coherence length at zero temperature deduced from $B_{\mathrm{c} 2}$ in RM and CP experiments were $\xi_{0}=1.97 \mathrm{~nm}$ and $2.13 \mathrm{~nm}$, respectively, similar to those observed in YBCO. The calculation was made using $\kappa \approx 100$.

\section{Conclusion}

In summary, we measured the electronic specific heat by a differential calorimetry in temperature range $74 \mathrm{~K}$ to $99 \mathrm{~K}$ and inferred the temperature dependence of reversible magnetization, $M_{\text {rev }}$, from the data. This procedure was confronted with a direct measurement of $M_{\text {rev }}$ made by SQUID. Both experiments gave results similar to those commonly observed in $\mathrm{YBa}_{2} \mathrm{Cu}_{3} \mathrm{O}_{7}$ (YBCO).

\section{Acknowledgments}

The authors acknowledge support from the grant ESO MNT-ERA (ME10069 of MEYS CR) and from the Grant Agency of the Czech Technical University in Prague, grant SGS10/296/OHK4/3T/14.

\section{References}

[1] M. Jirsa, M. Rameš, K. Jurek, M. Muralidhar, P. Das, M.R. Koblischka, T. Wolf, Mater. Sci. Engin. B 151, 25 (2008).

[2] W.Y. Liang, J.W. Loram, Physica $C$ 404, 230 (2004) or A.M. Campbell, J.W. Loram, N. Hari-Babu, J.R. Cooper, D.A. Cardwell, Phys. Rev. B 70, 214518 (2004). 